\title{
Intraoperative Neurophysiologic Assessment in Deep Brain Stimulation Surgery and its Impact on Lead Placement
}

\author{
Philipp Krauss $^{1}$ Markus Florian Oertel ${ }^{2}$ Heide Baumann-Vogel ${ }^{3}$ Lukas Imbach ${ }^{3}$ \\ Christian Rainer Baumann ${ }^{3}$ Johannes Sarnthein ${ }^{2} \quad$ Luca Regli ${ }^{2}$ Lennart Henning Stieglitz ${ }^{2}$ \\ ${ }^{1}$ Department of Neurosurgery, Klinikum rechts der Isar, Technical \\ University of Munich, Germany \\ ${ }^{2}$ Department of Neurosurgery, University Hospital Zurich, University \\ of Zurich, Zurich, Switzerland \\ ${ }^{3}$ Department of Neurology, University Hospital Zurich, University of \\ Zurich, Zurich, Switzerland \\ Address for correspondence Philipp Krauss, Department of \\ Neurosurgery, Klinikum rechts der Isar, Technical University of \\ Munich, Ismaninger Strasse 22, Munich 81675, Germany \\ (e-mail: ph.krauss@gmx.de). \\ J Neurol Surg A 2021;82:18-26.
}

\begin{abstract}
Objectives While the efficacy of deep brain stimulation (DBS) to treat various neurological disorders is undisputed, the surgical methods differ widely and the importance of intraoperative microelectrode recording (MER) or macrostimulation (MS) remains controversially debated. The objective of this study is to evaluate the impact of MER and MS on intraoperative lead placement.

Patients and Methods We included 101 patients who underwent awake bilateral implantation of electrodes in the subthalamic nucleus with MER and MS for Parkinson's disease from 2009 to 2017 in a retrospective observational study. We analyzed intraoperative motor outcomes between anatomically planned stimulation point (PSP) and definite stimulation point (DSP), lead adjustments and Unified Parkinson's Disease Rating Scale Item III (UPDRS-III), levodopa equivalent daily dose (LEDD), and adverse events (AE) after 6 months.

Results We adjusted 65/202 leads in 47/101 patients. In adjusted leads, MS results improved significantly when comparing PSP and DSP $(p<0.001)$, resulting in a number needed to treat of 9.6. After DBS, UPDRS-III and LEDD improved significantly after

Keywords

- DBS

- deep brain stimulation

- intraoperative neurophysiology

- neuromodulation

- Parkinson's disease 6 months in adjusted and nonadjusted patients $(p<0.001)$. In $87 \%$ of leads, the active contact at 6 months still covered the optimal stimulation point during surgery. In total, $15 \mathrm{AE}$ occurred.

Conclusion MER and MS have a relevant impact on the intraoperative decision of final lead placement and prevent from a substantial rate of poor stimulation outcome. The optimal stimulation points during surgery and chronic stimulation strongly overlap. Follow-up UPDRS-III results, LEDD reductions, and DBS-related AE correspond well to previously published data.
\end{abstract}

received

October 12, 2019

accepted after revision

February 13, 2020

published online

October 13, 2020 (c) 2020 Georg Thieme Verlag KG

Stuttgart - New York
DOI https://doi.org/

10.1055/s-0040-1716329. ISSN 2193-6315. 


\section{Introduction}

Deep brain stimulation (DBS) is a well-accepted and highly effective surgical treatment option for diverse neurological and psychiatric disorders. ${ }^{1-8}$ The widest experience in DBS exists in movement disorders ${ }^{3,6}$ including Parkinson's disease (PD), ${ }^{1,5,7,8}$ with significant improvement by DBS being shown in various clinical trials.

The anatomical targets are usually small and deep seated, and the precise determination of the stimulation point is of utmost importance to optimize clinical outcome and minimize stimulation related side effects (SRSE). Despite the widespread use of DBS, techniques of intracranial lead placement differ widely among functional neurosurgeons. ${ }^{9}$ Some centers perform electrophysiologic evaluation of the target region by microelectrode recording (MER). ${ }^{10-12}$ Additionally, intraoperative macrostimulation (MS) during awake surgery can provide direct feedback on stimulation efficacy and potential SRSE. ${ }^{13}$

However, multiple brain penetrations and MER might raise the risk of intracranial bleeding. ${ }^{14}$ Furthermore, the duration and costs of surgery can increase with the use of additional intraoperative tools. ${ }^{15}$ Although some authors reported good clinical results by magnetic resonance imaging (MRI) based targeting and lead verification alone, ${ }^{16-19}$ DBS surgery without MS or MER will always leave the probing question of if intraoperative neurophysiology would have resulted in optimization of target sites despite stereotactic accuracy.

In this study, we therefore set out to determine the impact of neurophysiologic evaluation for awake surgery compared with exclusively anatomical and image-guided targeting in patients with PD who underwent bilateral subthalamic nucleus (STN) DBS based on the following outcome measures: intraoperative motor outcome between the anatomically planned stimulation point (PSP) and the definite stimulation point (DSP), rates of intraoperative lead adjustment, as well as changes in Unified PD Rating Scale item III (UPDRS III), levodopa equivalent daily dose (LEDD), and adverse events (AE) during a 6-months follow-up.

\section{Patients and Methods}

\section{Study Design}

We performed an observational retrospective study by analysis of prospectively acquired patient-specific datasets documented in our clinical database. All data were collected, encrypted, processed, and analyzed according to the study protocol approved by the local ethics committee (KEK-ZH: ID 2017-00440, version 1.2). All the included patients gave their written consent.

\section{Patient Selection}

All the patients with confirmed diagnosis of PD responding to levodopa, disabling motor fluctuations, or severe functional impairment despite best medical therapy (BMT) who underwent awake bilateral implantation of a DBS system in the STN between April 2009 and February 2017 and completed a clinical follow-up of at least 6 months were included. Patients were selected for DBS by an interdisciplinary board of neurosurgeons and neurologists according to the guideline of the German, Austrian, and Swiss neurological societies for the diagnosis and treatment of PD. ${ }^{20}$ Absence of contraindications such as severe neuropsychological or psychiatric impairment, intracranial mass lesions, coagulopathy, or relevant anesthesiologic perioperative risk constellation was mandatory.

\section{Neurological Evaluation}

A movement disorder-specialized neurologist performed the UPDRS III $^{21}$ before DBS under BMT ("pre-DBS ON/OFF"), under complete withdrawal of anti-Parkinson's medication ("pre-DBS OFF/OFF"), during a structured levodopa responsiveness test ("levodopa Test ON/OFF"), and after DBS plus BMT ("post-DBS ON/ON"). ${ }^{22}$ Evaluation after DBS surgery in an "OFF/ON" condition was not performed, as the follow-up paradigm was designed to fulfill clinical purposes. We calculated an LEDD pre- and post-DBS and registered SRSE during surgery and until the postoperative follow-up of a minimum of 6 months. ${ }^{23}$ The postoperative stimulation paradigm was defined according to the patient's individual clinical response along with LEDD reduction over several weeks in a nonstandardized way. Stimulation voltage was slowly increased during rehabilitation stay and short-term follow-up consultations or by the patient themself within predefined ranges. Stimulation contacts or parameters were adjusted if intolerable side effects or insufficient stimulation response occurred based on individual neurological evaluation, respectively. The initial settings consisted of monopolar stimulation at the intraoperatively identified optimal contact.

\section{Stereotactic Planning}

Stereotactic targeting of the posterolateral STN was performed using the StealthStation FrameLink planning software and Cranial 3 planning software (Medtronic, Minneapolis, Minnesota, United States) based on patient-specific cranial MRI 3D space $\mathrm{T} 2$ and 3D space $\mathrm{T} 1$ gadolinium-enhanced sequences (3-T Skyra VD13, Siemens Healthineers GmbH, Erlangen, Germany). The MR planning images were fused to a frame-based stereotactic computed tomography (CT; 1-mm slice thickness with contrast agent; SOMATOM Sensation 64, after June 2016 SOMATOM Definition AS, Siemens Healthineers GmbH, Erlangen, Germany) on the day of surgery. The PSP was regularly defined as $2 \mathrm{~mm}$ inferior of the electrophysiologically identified STN entry point. Immediately after lead insertion, a native CT with the stereotactic frame was performed and fused to the initial planning CT. The Euclidian distance between the intended (including intraoperative adjustment) and the actual lead trajectory was calculated for anteroposterior (AP) and mediolateral (ML) coordinates using the center of the lead artifact in the probe's eye view to determine the actual lead. ${ }^{24}$ The depth was regularly defined according to MER findings. If present, lateral lead aberrance of $\geq 2 \mathrm{~mm}$ was corrected during the same surgical session.

\section{Surgical Protocol and Microelectrode Recording}

All lead implantations were performed awake under local anesthesia as previously described using a Riechert- 
Mundinger stereotactic frame (Inomed, Emmendingen, Germany) and CT registration. ${ }^{25}$ MER was performed in $0.5-\mathrm{mm}$ steps starting at $10 \mathrm{~mm}$ above the planned target via a single central electrode (FHC Inc., Bowdoin, Maine, United States). Thereafter, MS was performed at the PSP $(0-4 \mathrm{~mA}$ in $1-\mathrm{mA}$ steps at $130 \mathrm{~Hz}$ and a pulse width of $60 \mu \mathrm{s})$ along with clinical assessment. We adjusted the PSP in depth or by adding $2 \mathrm{~mm}$ parallel trajectories via a five-position Ben-Gun array only if the MER was not detecting characteristic STN signals or if the MS showed insufficient stimulation responses as described later. ${ }^{26}$ This final adjusted stimulation point was referred to as the DSP. Finally, the four-contact intracranial lead (model 3389, Medtronic, Minneapolis, Minnesota, United States) was implanted under fluoroscopic guidance.

\section{Intraoperative Clinical Assessment and Follow-up}

During surgery, upper limb rigidity $(\mathrm{R})$, akinesia $(\mathrm{A})$, and tremor $(T)$ were examined corresponding to the UPDRS III by a neurologist. The response to stimulation was expressed relatively to the symptom improvement during the levodopa test ("pre-DBS OFF/OFF-" - "levodopa test ON/OFF" $=100 \%$ ). To address different PD phenotypes, these results were weighted for analysis as follows: (RAT-protocol): akinetic/rigid (outcome $=R+A / 2$ ), equivalent (outcome $=\mathrm{R}+\mathrm{A}+\mathrm{T} / 3$ ), tremor (outcome $=\mathrm{T}$ ). Response to stimulation was regarded optimal with a relative response of $\geq 75 \%$ without SRSE. Occurrence of side effects at $\leq 2 \mathrm{~mA}$ was regarded as too narrow a therapeutic window. Surgeryor stimulation-related $\mathrm{AE}$ were registered from the day of surgery up to the 6-month postoperative follow-up. Surgical site infections were registered up to 90 days after surgery according to the Centers for Disease Control and Prevention guidelines. ${ }^{27}$

\section{Data Analysis and Statistics}

Statistical analysis was performed using the SPSS statistics software (V.24, IBM, Armonk, New York, United States). Data sets were tested for normal distribution according to the central limit theorem and the Kolmogorov-Smirnov test. A $p$ value $\leq 0.05$ was considered significant and indicated by “*," $p$ values $\leq 0.01$ were indicated by “**," and $p$ values $\leq 0.001$ by “***." Paired or unpaired two-tailed Student's $t$-test was performed, or a nonparametric equivalent was used, if the prerequisites for parametric testing were not met. For correlating data (such as complications, surgery durations, and MS sites), the linear regression was used. Data in text and graphs are shown as mean \pm standard deviation (SD) or median (mdn) with interquartile range (IQR), respectively, with Pearson's effect size (r), if not indicated otherwise.

\section{Results}

\section{Study Population}

Of 187 patients who received DBS surgery and completed a 6-month follow-up, 101 patients fulfilled all criteria and were included in the study (-Table 1, - Fig. $\mathbf{1}$ ).
Table 1 Patient properties

\begin{tabular}{|l|l|}
\hline Sex (M: F) & $59: 42$ \\
\hline Surgeon (LS: OS: LS/OS) & $46: 52: 3$ \\
\hline Mean age $(\mathrm{y} \pm$ SD) & $62.4 \pm 9.2$ y \\
\hline Mean disease duration $(\mathrm{y} \pm \mathrm{SD})$ & $11.4 \pm 5.2 \mathrm{y}$ \\
\hline Phenotype (AKIN: TREM: EQV) & $54: 25: 22$ \\
\hline
\end{tabular}

Abbreviations: AKIN, akinetic; EQV, equivalent; F, female; LHS, Lennart Henning Stieglitz; M, male; OS, Oguzkan Sürücü; SD, standard deviation; TREM, tremor; $y$, year.

\section{All patients implanted $(n=187$}

\section{Patients included $(n=101)$}

Fig. 1 Patient inclusion process. (n, number; PD, Parkinson's disease; STN, subthalamic nucleus; GA, general anesthesia.)

\section{Lead Adjustments}

Forty-nine percent of patients received unilateral (31/101) or bilateral (17/101) intraoperative lead adjustment. Out of 202 implanted electrodes, 65 were adjusted following the results of either MS (59) or MER (6). These leads were adjusted as follows: medial: $n=27$; posterior: $n=5$; lateral: $n=4$; anterior: $n=4$; and central depth trajectory adjustment: $n=25$.

\section{Stereotactic Accuracy}

Euclidian error between the calculated (including adjustment) and the actual lead location was $0.87 \mathrm{~mm} \pm 0.61 \mathrm{SD}$. Adjusted leads showed a slightly higher error than nonadjusted leads (nonadjusted lead: $0.79 \mathrm{~mm} \pm 0.57 \mathrm{SD}$ vs. adjusted leads $1.04 \mathrm{~mm} \pm 0.64 \mathrm{SD}$ ). Five leads required adjustment due to stereotactic inaccuracy $\geq 2 \mathrm{~mm}$ (two nonadjusted vs. three adjusted) detected by intraoperative control CT. This was followed by lead repositioning, including neurophysiologic reassessment, and verification of stereotactic accuracy by intraoperative CT was performed during the same operation. We assumed lead malposition after replacement of the rigid macroelectrode by the more flexible permanent lead as the cause of reintervention in these cases.

\section{Influence of Lead Adjustment on Intraoperative Results} Patient phenotype adapted intraoperative clinical response in the absence of SRSE was grouped as follows: optimal $\geq 75 \%$, good $74.9 \%$ - 50\%, intermediate $49.9 \%$ - $25 \%$, and insufficient 


\title{
Intraoperative RAT grouped
}

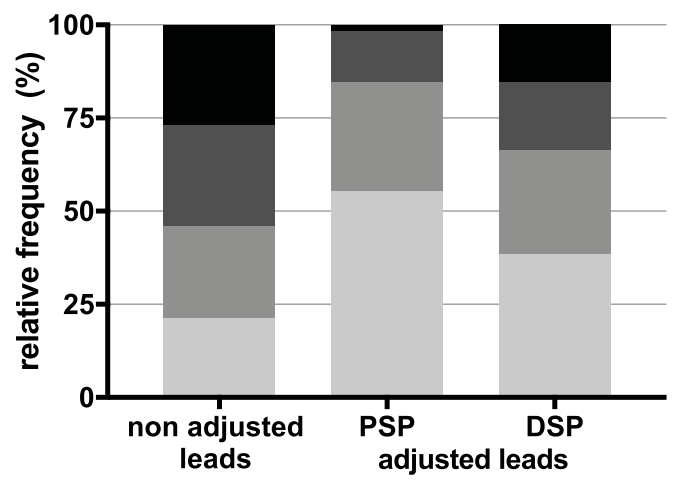

\author{
levodopa test \\ response \% \\ $\geq 75 \%$ \\ $74.9 \% \leq 50 \%$ \\ $49.9 \% \leq 25 \%$ \\ $<25 \%$
}

Fig. 2 Intraoperative RAT grouped. Relative frequencies of response groups per lead ( $\geq 75 \%, 74.9-50 \%, 49.9-25 \%$, and $<25 \%$ ) in nonadjusted (27, 27, 24.8, and 21.2\%), adjusted PSP (1.5, 13.8, 29.2, and 55.4\%), and adjusted DSP (15.4, 18.5, 27.7, and 38.5\%). (RAT, RAT protocol; PSP, planned stimulation point; DSP, definite stimulation point.)

$<25 \%$. In the nonadjusted group, 37/137 leads showed optimal results and 29/137 showed insufficient results. In the adjusted group, 1/65 showed optimal results and 36/65 showed insufficient outcome before adjustment (PSP) compared with 10/65 optimal and 25/65 insufficient outcomes after adjustment (DSP). The overall variance of distribution between PSP and DSP of adjusted leads differed significantly with a shift toward better response in the DSP group (chi-square [64] $=240.02$, $p<0.001, n=65$; - Fig. 2). The variance between DSP of adjusted leads and the nonadjusted leads reached no significant difference (chi-square $[64]=61.69, p>0.05, n=65$; -Fig. 2). The median response of the actually adjusted leads improved significantly (PSP mdn 0.0\% [IQR 0.0-35.4\%] vs. DSP mdn 25.0\% [IQR 0.0-50\%]; asymptotic Wilcoxon signed-rank test: $p<0.001, n=65, r=0.43$ ) but did not reach the efficacy of leads without adjustment of the initial position (nonadjusted leads mdn 50.0\% [IQR 25.0-75.0\%] vs. adjusted leads DSP mdn 25.0\% [IQR 0.0-50.0\%]; two-tailed Mann-Whitney $U$ test: $U=3,251.000, p<0.01, n=202, r=0.22$; - Fig. 3). The DSP showed a better response than the PSP in 25/65 of the adjusted

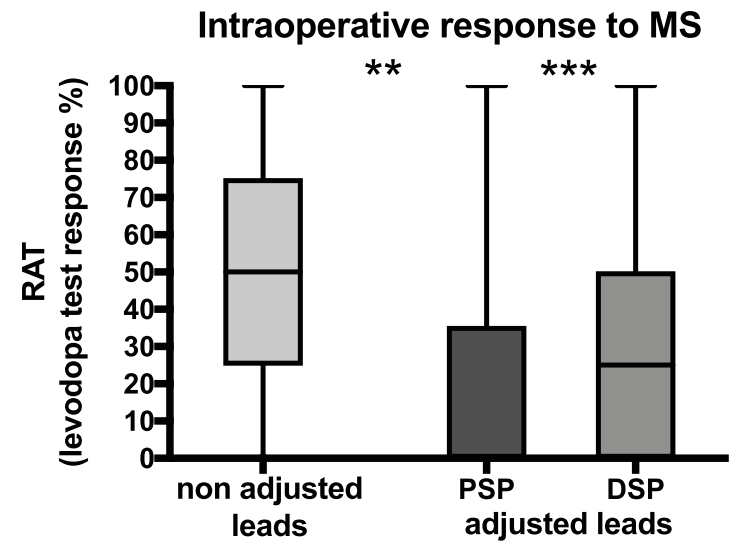

Fig. 3 Intraoperative response to MS. Relative intraoperative clinical improvement to test stimulation referenced to the levodopa responsiveness test outcome according to the RAT protocol. Data displayed grouped as mdn (IQR). (“**” $=p<0.01$; “***” $=p<0.001$; IQR, interquartile range; mdn, median; MS, macrostimulation; RAT, RAT protocol; $n$, number; PSP, planned stimulation point; DSP, definite stimulation point.) leads, an indifferent response in 36/65 leads, and a less effective response in 4/65 leads according to the criteria described earlier. This resulted in a number needed to treat (NNT) of 9.6 per electrode. Mean stimulation current without SRSE did not differ significantly between DSP and nonadjusted leads (nonadjusted $1.79 \mathrm{~mA} \pm 1.11 \mathrm{SD}, \quad n=137$ vs. DSP $1.92 \mathrm{~mA} \pm 1.39 \mathrm{SD}, n=65$; two-tailed independent Student's $t$-test: $t(200)=-0.7, p=0.49,95 \%$ confidence interval $[\mathrm{CI}]$ $(-0.4851: 0.231), r=0.05)$, but was significantly higher than in the PSP (DSP $1.92 \mathrm{~mA} \pm 1.39$ SD vs. PSP $1.38 \mathrm{~mA} \pm 1.23 \mathrm{SD}$; two-tailed paired samples Student's $t$-test: $t(64)=-3.152$, $p<0.01,95 \%$ CI $(-0.8671:-0.1944), r=0.37)$.

\section{Motor Outcome and Levodopa Equivalent Daily Dose}

The first regular follow-up was after $203 \pm 43$ SD days postoperatively. The UPDRS III score during "post-DBS ON/ON" compared with "pre-DBS ON/OFF" and "levodopa Test ON/OFF" improved significantly in all groups (-Table 2, - Fig. 4). The UPDRS III scores did not differ significantly between groups at any time point (statistics not shown). The LEDD decreased significantly after DBS in all groups (-Table 2, - Fig. 5), with a slightly higher LEDD during the "pre-DBS ON/OFF" and higher absolute levodopa reduction (-Table 2 ) in the nonadjusted group. The "post-DBS ON/ON" LEDD did not differ significantly between adjusted and nonadjusted lead patients (statistics not shown). DBS parameters at the follow-up showed 155/202 monopolar, 44/202 bipolar, and 3/202 interleaved mode configurations ( $\varnothing$ amplitude: $2.85 \mathrm{~V} \pm 0.79 \mathrm{SD}$; $\varnothing$ pulse width of $60 \pm 4.23 \mathrm{SD} \mathrm{ms}$; and $\varnothing$ frequency $132.92 \mathrm{~Hz} \pm 11.11 \mathrm{SD}$ ). In $175 / 202$ electrodes (56/65 in the adjusted and 119/137 in the nonadjusted group), the active stimulation contacts after 6 months covered the site of optimal intraoperative stimulation. The adjusted leads reached a slightly higher stimulation voltage than the nonadjusted leads (adjusted $3.0 \mathrm{~V} \pm 0.8 \mathrm{SD}, n=65 \mathrm{vs}$. nonadjusted $2.8 \mathrm{~V} \pm 0.8 \mathrm{SD}, n=137$; two-tailed independent Student's $t$-test; $t(200)=-2.407, p=0.17, r=0.17)$.

\section{Complications}

Stimulation- or surgery-related AE occurred in 15/101 patients including 1 cardiopulmonary decompensation, 
Table 2 Patient outcome

\begin{tabular}{|c|c|c|c|}
\hline Patient outcome & All patients $(n=101)$ & Adjusted patients $(n=54)$ & Nonadjusted patients $(n=47)$ \\
\hline UPDRS III pre-DBS ON/OFF & $22.0(13.5-31.5) \mathrm{pt}$. & $22.0(16.0-31.0)$ pt. & $21.5(13.0-32.0) \mathrm{pt}$. \\
\hline UPDRS III pre-DBS OFF/OFF & $40.0(28.0-50.0)$ pt. & $42.0(30.0-52.0) \mathrm{pt}$. & $36.5(26.0-46.5) \mathrm{pt}$. \\
\hline $\begin{array}{l}\text { UPDRS III levodopa } \\
\text { test ON/OFF }\end{array}$ & $18.0(12.0-23.0) \mathrm{pt}$. & $18.0(12.0-24.0) \mathrm{pt}$. & $18.5(13.0-23.0) \mathrm{pt}$. \\
\hline UPDRS III post-DBS ON/ON & $15.0(9.0-19.0) \mathrm{pt}$. & $15.0(9.0-19.0) \mathrm{pt}$. & $16.5(9.0-19.8) \mathrm{pt}$. \\
\hline $\begin{array}{l}\text { Difference UPDRS III post-DBS } \\
\text { ON/ON - pre-DBS ON/OFF }\end{array}$ & $9.0(4.0-15.0) \mathrm{pt}$. & $8.0(4.0-15.0) \mathrm{pt}$. & $9.0(4.0-16.3) \mathrm{pt}$. \\
\hline LEDD pre-DBS ON/OFF & $1160.0(862.5-1588.1) \mathrm{mg} / \mathrm{d}$ & $1025.0(778.0-1411.5) \mathrm{mg} / \mathrm{d}^{*}$ & $1318.8(1000.0-1741.5) \mathrm{mg} / \mathrm{d}^{*}$ \\
\hline LEDD post-DBS ON/ON & $399.3(187.5-730.1) \mathrm{mg} / \mathrm{d}$ & $337.5(120.0-637.5) \mathrm{mg} / \mathrm{d}$ & $468.8(200.0-789.375) \mathrm{mg} / \mathrm{d}$ \\
\hline $\begin{array}{l}\text { Difference LEDD post-DBS } \\
\text { ON/ON - pre-DBS ON/OFF }\end{array}$ & $765.5(376.5-1057.8) \mathrm{mg} / \mathrm{d}$ & $639.5(250.0-957.5) \mathrm{mg} / \mathrm{d}^{*}$ & $834.4(564.6-1155.6) \mathrm{mg} / \mathrm{d}^{*}$ \\
\hline
\end{tabular}

Abbreviations: DBS, deep brain stimulation; LEDD, levodopa equivalent daily dose; pt., points; UPDRS III, Unified Parkinson's disease rating scale item III. Note: The outcome showed no significant intergroup difference at any time point despite values indicated by a *. Adjusted patients ( $n=47$ ) versus nonadjusted patients ( $n=54$ ), LEDD pre-DBS ON/OFF (two-tailed Mann-Whitney $U$ test: $U=919.000, p<0.05 ; r=0.24$ ), difference LEDD post-DBS ON/ON - pre-DBS ON/OFF (two-tailed Mann-Whitney $U$ test: $U=978.000, p<0.05 ; r=0.19$ ).

Data displayed as median with interquartile range ( $\mathrm{mdn}(\mathrm{IQR}))$ rounded to one decimal.

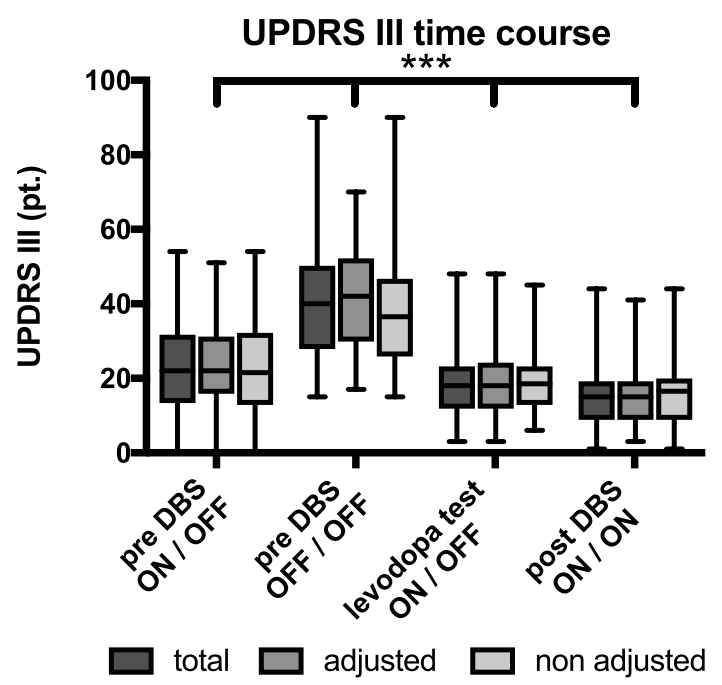

Fig. 4 Motor outcome time course. Significance of difference between time points: asymptotic Wilcoxon signed-rank test-all patients $(n=101)$ : pre-DBS ON/OFF versus pre-DBS OFF/OFF: $p<0.001, r=0.83$; versus levodopa test ON/OFF: $p<0.001, r=0.45$; versus post-DBS ON/ON: $p<0.001, r=0.59$; pre-DBS OFF/OFF versus levodopa test ON/OFF: $p<0.001, r=0.86$; versus post-DBS ON/ON: $p<0.001, r=0.86$; levodopa test ON/OFF versus post-DBS ON/ON: $p<0.001, r=0.38$; adjusted patients $(n=47)$ : pre-DBS ON/OFF versus pre-DBS OFF/OFF: $p<0.001, r=0.83$; versus levodopa test ON/OFF: $p=0.002, r=0.46$; versus post-DBS ON/ON: $p<0.001, r=0.65$; pre-DBS OFF/OFF versus levodopa test ON/OFF: $p<0.001, r=0.86$; versus post-DBS ON/ON: $p<0.001, r=0.87$; levodopa test ON/OFF versus post-DBS ON/ON: $p<0.01, r=0.45$; and nonadjusted patients $(n=54)$ : pre-DBS ON/OFF versus pre-DBS OFF/OFF: $p<0.001$, $r=0.83$; versus levodopa test ON/OFF: $p=0.001, r=0.44$; versus post-DBS ON/ON: $p<0.001, r=0.54$; pre-DBS OFF/OFF versus levodopa test ON/OFF: $p<0.001, r=0.87$; versus post-DBS ON/ON: $p<0.001, r=0.85$; levodopa test ON/OFF versus post-DBS ON/ON: $p<0.02, r=0.32$. Data displayed as $\mathrm{mdn}(\mathrm{IQR})$. (“**** $=p<0.001$; IQR, interquartile range; $\mathrm{mdn}$, median; UPDRS III, Unified Parkinson's Disease Rating Scale Item III; pt., points; DBS, deep brain stimulation; n, number.)

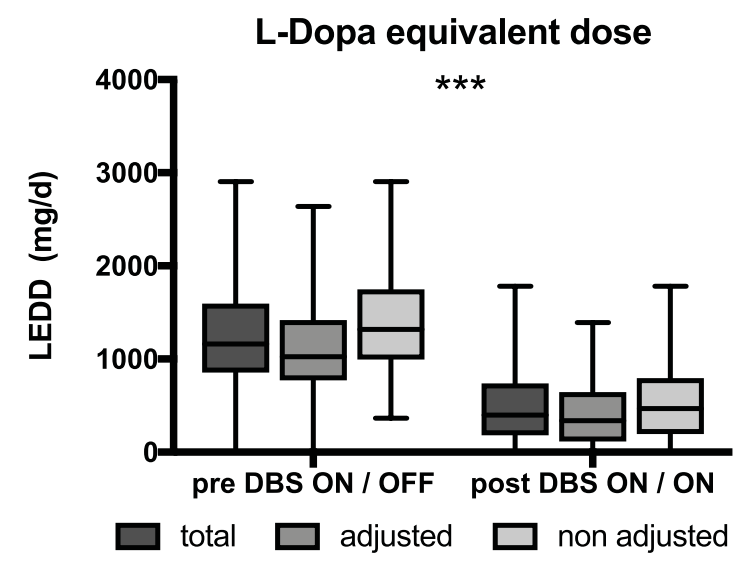

Fig. 5 LEDD time course. Significance of difference between time points: symptomatic Wilcoxon signed-rank test: all patients $(n=101)$ : $p<0.001, r=0.83$; adjusted patients $(n=47): p<0.001, r=0.84$; nonadjusted patients $(n=54): p<0.001$; data displayed as $\mathrm{mdn}$ $(\mathrm{IQR}) ; r=0.82$. ( “***” $=p<0.001 ; \mathrm{IQR}$, interquartile range; $\mathrm{mdn}$, median; LEDD, levodopa equivalent daily dose; mg, milligram; d, day; DBS, deep brain stimulation; $n$, number.)

2 hardware failures, 3 asymptomatic and 1 symptomatic intracranial hemorrhage, 3 internal pulse generator infections, 1 transient hypomania, 1 excessive day time sleepiness, 1 major dysarthria, 1 eyelid apraxia, and 1 memory decline. There was no intergroup difference for AE according to surgery duration (complications mdn 260 [IQR 237.5-290.0] minutes vs. no complications mdn 290 [IQR 255-350] minutes; two-tailed Mann-Whitney $U$ test; $U=518.000, p=0.23, n=101, r=0.12$ ) or number of MS sites (complications mdn 4 [IQR 2-5] sites vs. no complications mdn 3 [IQR 2-5] sites; two-tailed Mann-Whitney $U$ test; $U=620.000, p=0.81, n=101, r=0.02$; - Table 3). 
Table 3 Perioperative complications

\begin{tabular}{|l|l|l|l|l|}
\hline \multicolumn{5}{|l|}{ Perioperative complications } \\
\hline $\begin{array}{l}\text { MS } \\
(\mathbf{n}\end{array}$ & $\begin{array}{l}\text { Patients } \\
(\boldsymbol{n})\end{array}$ & $\begin{array}{l}\text { Mean surgery } \\
\text { duration } \\
(\min \pm \text { SD) }\end{array}$ & $\mathrm{AE}(\boldsymbol{n})$ & $\begin{array}{l}\text { AE/patients } \\
(\boldsymbol{n} / \mathbf{n})\end{array}$ \\
\hline 2 & 42 & $257 \pm 44.8$ & 7 & 0.17 \\
\hline 3 & 10 & $312.5 \pm 86.9$ & 0 & - \\
\hline 4 & 16 & $330.3 \pm 116.5$ & 3 & 0.19 \\
\hline 5 & 9 & $332.8 \pm 69.5$ & 2 & 0.22 \\
\hline 6 & 9 & $341.7 \pm 56.8$ & 2 & 0.22 \\
\hline 7 & 10 & $387 \pm 72.1$ & 0 & - \\
\hline 8 & 1 & 285 & 0 & - \\
\hline 9 & 1 & 295 & 1 & 1 \\
\hline 10 & 2 & $622.5 \pm 286.4$ & 0 & - \\
\hline 11 & 0 & - & 0 & - \\
\hline 12 & 1 & 510 & 0 & - \\
\hline Total & 101 & $311.7 \pm 99$ & 15 & 0.15 \\
\hline
\end{tabular}

Abbreviations: AE, adverse events; MS, macrostimulation; SD, standard deviation.

Note: The number of MS prolonged the mean surgery duration, but was not significantly associated with more AE.

Comparing AE in patients who had two MS with patients who had multiple MS resulted in a number needed to harm of 294.1. Surgery duration was significantly correlated with the number of MS sites $(F(1.99)=50.106$, $p<0.001$ ) with every MS site prolonging surgery by 26 minute mean.

\section{Discussion}

\section{Intraoperative Lead Adjustment}

In DBS surgery, precise targeting is one of the key steps to success, and modifications after the final lead placement are limited. ${ }^{28,29}$ Despite stereotactic precision, MER and MS are widely used tools to optimize intraoperative lead placement. However, these techniques might also influence the duration, costs, and risks of the procedure.

In our cohort, with a high accuracy of stereotactic lead placement, we performed intraoperative lead adjustment in 47/101 patients and 65/202 implanted leads based on intraoperative neurophysiologic methods. In the literature, adjustment rates of up to $53 \%$ due to MER and MS results and of $25 \%$ when exclusively using an awake surgery approach with MS have been reported. ${ }^{10,30}$ Lower rates are described for "asleep" solely image-based DBS with only 4 of 70 leads repositioned due to lead deviation of $\geq 2 \mathrm{~mm}$ from the planned target. ${ }^{18}$ Clearly, the more information has to be incorporated the more lead adjustments might be necessary and merely the adjustment rates do not represent a beneficial value by itself. These rates reflect an important impact of MER and MS results on how surgery is performed and how they could prevent possible errors of our surgical strategy.

\section{Intraoperative Neurophysiologic Recording}

In our cohort, only 3\% of the leads were directly adjusted based solely on poor MER results without further MS. Other authors report an impact of MER of up to $35 \%$ on adjustment rates. ${ }^{12,31}$ MER may have its advantages in identifying the electrophysiologic footprint of some target structures ${ }^{11}$ that can differ from the MRI-derived target boundaries and can be performed under general anesthesia or dopaminergic medication. ${ }^{24,32,33}$ The widespread rates of MER-based adjustments in the literature may be explained by the variable a priori use of single or multiple microelectrodes. ${ }^{11}$ Often, more than one, for example, two to three microelectrodes are used in a classical neurophysiologic mapping approach. The low number of adjustments exclusively based on MER results in our cohort may be explained by the fact that we performed a single-trajectory MER in every patient from the beginning but adding them on demand. Therefore, MER was used more as a targeting control than a proper mapping tool.

\section{Intraoperative Macrostimulation}

MS played a profound role in our cohort, leading to adjustments in $29 \%$ of all electrodes. As we targeted the posterolateral part of the STN, capsular side effects may explain the number of medial corrections and possibly of the need of higher stimulation current after correction. Given the inherently incomplete intraoperative clinical assessment and the sometimes-delayed stimulation effect, the absence of SRSE presents a major outcome parameter of intraoperative MS. This represents an NNT of 9.6 for the whole cohort to benefit from the combined use of MER and MS. We admit that a microlesioning effect can blur the intraoperative response to side effects ratio. However, a current-dependent response pattern is suggestive of an actual stimulation effect on clinical improvement. In our cohort, a reduced outcome at the adjusted stimulation point in some leads may seem irritating and reflects the method of analysis of intraoperative data in this report. When looking on these selected cases in more detail, the DSP had better scoring results compared with the PSP at higher amplitude levels with minor SRSE, explaining the intraoperative decision to place the lead at the given stimulation point (data not shown). Many neurosurgeons rely on the results of MS to optimize the lead location during surgery although its predictive value is not fully clarified to date. ${ }^{34}$ Discrepancies between test and definite stimulation outcome may be due to different electrical properties of stimulation leads and the stimulated tissue. ${ }^{35}$ On the other hand, sparing intraoperative testing and relying only on imageverified implantation may lead to nonoptimal results as the MRI-defined optimal target location was found to differ by up to $1.4 \mathrm{~mm} .{ }^{36}$ Whether these findings and the high adjustment rates in neurophysiologic based lead implantations represent mainly a result of insufficient targeting including stereotactic inaccuracy due to CT and MRI fusion error cannot be finally ruled out, as postoperative MRI scans were not realized in our cohort. ${ }^{37}$ However, it has been shown that high stereotactic precision without fusion error can be achieved using a planning MRI with adjustment protocols. ${ }^{38}$ This technique has been reported to result in equal long-term efficacy both for awake 
surgery with MS and asleep surgery protocols with the limitation that adjustment rates due to intraoperative testing were not emphasized. ${ }^{19}$ If our findings would equally apply to exclusively MRI-based DBS finally cannot be answered. In this study, a stereotactic CT control was only used to identify potential cases of relevant stereotactic, more precisely, frame inaccuracy and verify or adjust them according to neurophysiologic reassessment. In our experience, MER and, with an even higher impact, MS seem to represent viable tools to refine intraoperative lead placement that could also be caused by inaccurate targeting and possible fusion error.

\section{Postoperative Outcome}

The clinical outcome in our cohort is comparable or slightly better compared with results of most previous studies $1,5,7,8$ with a significant reduction of the LEDD and UPDRS III improvement and might be even superior compared with smaller cohorts undergoing asleep DBS without MER or MS, if considering only the stimulation ON/BMT ON condition. ${ }^{16,19}$ The optimal intraoperative stimulation point was covered by the chronic stimulation in over $85 \%$ of cases, underlying the relevance of the intraoperative information. However, the postoperative stimulation paradigm was defined in a nonstandardized way and no significant correlation between intraoperative clinical response and LEDD or UPDRS III outcome was found in our cohort (data not shown). A possible predictive value may be masked by the strict protocol of analysis in our report as mentioned earlier (see the "Results" and "Intraoperative macrostimulation" sections). Nevertheless, MS helps in avoiding stimulation points with low SRSE thresholds that may hinder beneficial effects, which can only be seen in more complete and longterm clinical assessments.

\section{Duration of Surgery and DBS-Related Adverse Events} Surgery duration (mean 312 minutes \pm 99 minute SD) correlated significantly with the number of MS sites and was longer compared with reported durations of asleep DBS. ${ }^{18,39}$ This could be particularly explained by the specific events defining the beginning and end of surgery at our department (including pre- and postoperative $\mathrm{CT}$ and transfer to the scanner outside the operation room). The AE rate per patient (15\%) did not correlate with surgery duration or MS sites and was similar to previously published data including a $2 \%$ rate of intracranial hemorrhage per lead. ${ }^{40}$ All intracranial hemorrhages occurred in patients with multiple brain penetrations $(4,5,6$, and 9 penetrations per patient), which is in accordance with prior reports. ${ }^{14}$ Two hemorrhages consisted of subarachnoid blood at the lead insertion zone, most likely caused by the surgical approach before electrode insertion. Only one deep hemorrhage was symptomatic, and the patient ecovered completely after 6 months from a transient paresis of the lower limb.

\section{Study Limitations}

We addressed the different PD phenotypes by pooling parts of the complete UPDRS III score for the cardinal symptoms and weighted those in a rather simple way. More detailed neurological assessments are hardly feasible during intraoperative routine, and the study's retrospective design did not allow predefining strict criteria for lead adjustment. Nevertheless, these data are of value as they reflect the information available in the intraoperative decision process. Motor outcome on chronic stimulation was analyzed in DBS plus BMT conditions, based on clinical purposes. This limits the comparison of exclusively stimulation-related effects between our approach and other studies, reporting results in DBS ON/BMT OFF conditions. Our outcome parameter has to be evaluated together with the changes in the LEDD, which can depend on the treating neurologist's regime or an aggravation of nonmotor symptoms by LEDD reduction. In this study, we were not able to address these questions, as structured assessments of AE such as UPDRS IV were not available for every patient. Nevertheless, the overall clinical improvement after DBS, including motor outcome and LEDD reduction, was very favorable in our cohort, suggesting an important effect of stimulation. The absence of a structured programming paradigm for post-DBS settings weakens the predictive value of the intraoperative identified stimulation contacts but reflects daily clinical practice, where DBS programming is individual and takes multiple factors into account. In a small number of leads, repositioning (including neurophysiologic reassessment) was performed due to gross deviations from the plan on the postoperative stereotactic CT scans. This may seem paradoxical. As we insert the definite lead along the micro-/macro-tract without guide cannula (only the semirigid mandrin), we claim a possible insertion error when exchanging micro/macro and definite lead to be responsible for this. In these leads, clinical, neurophysiologic and imaging results were as planned after adjustment. Finally, the outcome evaluation based on parameters that would not have been available without using the investigated electrophysiologic techniques represents an inherent source of error to our technique.

\section{Conclusion}

In our cohort of 101 patients undergoing bilateral STN DBS for PD, we could show a relevant impact of neurophysiologic evaluation on intraoperative decision-making regarding the final lead implantation. Adapting the lead location according to the results of MER and MS led to a significant improvement in the stimulation response compared with the initially planned stimulation targets. Especially initial poor responders improved up to the average of the cohort. Furthermore, the intraoperatively identified optimal stimulation sites highly matched with the chronically active contacts, suggesting a good prediction of optimal stimulation sites using these techniques. Using our surgical strategy, the clinical results of motor outcome and LEDD reduction were comparable to or even better than previously published data, but the results were similar regarding implantation-related risks for neurophysiologically verified DBS. In our experience, intraoperative neurophysiologic feedback helps find the optimal stimulation point and improve DBS surgery results. 


\section{Ethical Approval}

All procedures performed in this study were in accordance with the ethical standards of the regional research committee (Kantonale Ethikkomission Zürich) and the Declaration of Helsinki (1964) and its later amendments or comparable ethical standards. Informed consent was obtained from all individual participants included in the study.

\section{Conflict of Interest \\ None declared.}

\section{References}

1 Deuschl G, Schade-Brittinger C, Krack P, et al; German Parkinson Study Group, Neurostimulation Section. A randomized trial of deep-brain stimulation for Parkinson's disease. N Engl J Med 2006;355(09):896-908

2 Holtzheimer PE, Husain MM, Lisanby SH, et al. Subcallosal cingulate deep brain stimulation for treatment-resistant depression: a multisite, randomised, sham-controlled trial. Lancet Psychiatry 2017;4(11):839-849

3 Kupsch A, Benecke R, Müller J, et al; Deep-Brain Stimulation for Dystonia Study Group. Pallidal deep-brain stimulation in primary generalized or segmental dystonia. N Engl J Med 2006;355(19): 1978-1990

4 Mallet L, Polosan M, Jaafari N, et al; STOC Study Group. Subthalamic nucleus stimulation in severe obsessive-compulsive disorder. N Engl J Med 2008;359(20):2121-2134

5 Schuepbach WM, Rau J, Knudsen K, et al; EARLYSTIM Study Group. Neurostimulation for Parkinson's disease with early motor complications. N Engl J Med 2013;368(07):610-622

6 Schuurman PR, Bosch DA, Bossuyt PM, et al. A comparison of continuous thalamic stimulation and thalamotomy for suppression of severe tremor. N Engl J Med 2000;342(07): 461-468

7 Weaver FM, Follett K, Stern M, et al; CSP 468 Study Group. Bilateral deep brain stimulation vs best medical therapy for patients with advanced Parkinson disease: a randomized controlled trial. JAMA 2009;301(01):63-73

8 Williams A, Gill S, Varma T, et al; PD SURG Collaborative Group. Deep brain stimulation plus best medical therapy versus best medical therapy alone for advanced Parkinson's disease (PD SURG trial): a randomised, open-label trial. Lancet Neurol 2010;9(06): 581-591

9 Hamel W, Köppen JA, Alesch F, et al. Targeting of the subthalamic nucleus for deep brain stimulation: a survey among Parkinson disease specialists. World Neurosurg 2017;99:41-46

10 Bour LJ, Contarino MF, Foncke EM, et al. Long-term experience with intraoperative microrecording during DBS neurosurgery in STN and GPi. Acta Neurochir (Wien) 2010;152(12): 2069-2077

11 Gross RE, Krack P, Rodriguez-Oroz MC, Rezai AR, Benabid AL. Electrophysiological mapping for the implantation of deep brain stimulators for Parkinson's disease and tremor. Mov Disord 2006; 21(Suppl 14):S259-S283

12 Montgomery EB Jr. Microelectrode targeting of the subthalamic nucleus for deep brain stimulation surgery. Mov Disord 2012;27 (11):1387-1391

13 Machado A, Rezai AR, Kopell BH, Gross RE, Sharan AD, Benabid AL. Deep brain stimulation for Parkinson's disease: surgical technique and perioperative management. Mov Disord 2006;21 (Suppl 14):S247-S258

14 Zrinzo L, Foltynie T, Limousin P, Hariz MI. Reducing hemorrhagic complications in functional neurosurgery: a large case series and systematic literature review. J Neurosurg 2012;116(01):84-94
15 McClelland S III. A cost analysis of intraoperative microelectrode recording during subthalamic stimulation for Parkinson's disease. Mov Disord 2011;26(08):1422-1427

16 Aviles-Olmos I, Kefalopoulou Z, Tripoliti E, et al. Long-term outcome of subthalamic nucleus deep brain stimulation for Parkinson's disease using an MRI-guided and MRI-verified approach. J Neurol Neurosurg Psychiatry 2014;85(12):1419-1425

17 Brodsky MA, Anderson S, Murchison C, et al. Clinical outcomes of asleep vs awake deep brain stimulation for Parkinson disease. Neurology 2017;89(19):1944-1950

18 Mirzadeh Z, Chapple K, Lambert M, et al. Parkinson's disease outcomes after intraoperative CT-guided "asleep" deep brain stimulation in the globus pallidus internus. J Neurosurg 2016;124(04):902-907

19 Nakajima T, Zrinzo L, Foltynie T, et al. MRI-guided subthalamic nucleus deep brain stimulation without microelectrode recording: can we dispense with surgery under local anaesthesia? Stereotact Funct Neurosurg 2011;89(05):318-325

20 Deuschl G, Oertel W, Reichmann H. Leitlinien für Diagnostik und Therapie in der Neurologie: Idiopathisches Parkinson-Syndrom. Entwicklungsstufe: S3, Kurzversion. Aktualisierung 2016, AWMFRegister-Nummer: 030-010 Berlin: DGN (Hrsg); 2016

21 Fahn S, Elton RL. Unified Parkinson's Disease Rating Scale. In: Fahn S, Marsden CD, Goldstein M, Calne DB, eds. . Recent developments in Parkinson's disease. Florham Park, NJ: Macmillan Healthcare Information; 1987:153-163

22 Merello M, Nouzeilles MI, Arce GP, Leiguarda R. Accuracy of acute levodopa challenge for clinical prediction of sustained long-term levodopa response as a major criterion for idiopathic Parkinson's disease diagnosis. Mov Disord 2002;17(04):795-798

23 Tomlinson CL, Stowe R, Patel S, Rick C, Gray R, Clarke CE. Systematic review of levodopa dose equivalency reporting in Parkinson's disease. Mov Disord 2010;25(15):2649-2653

24 Nowacki A, Debove I, Fiechter M, et al. Targeting accuracy of the subthalamic nucleus in deep brain stimulation surgery: comparison between 3 T T2-weighted magnetic resonance imaging and microelectrode recording results. Oper Neurosurg (Hagerstown) 2018;15(01):66-71

25 Krauss P, Marahori NA, Oertel MF, Barth F, Stieglitz LH. Better hemodynamics and less antihypertensive medication: comparison of scalp block and local infiltration anesthesia for skull-pin placement in awake deep brain stimulation surgery. World Neurosurg 2018;120:e991-e999

26 Benazzouz A, Breit S, Koudsie A, Pollak P, Krack P, Benabid AL. Intraoperative microrecordings of the subthalamic nucleus in Parkinson's disease. Mov Disord 2002;17(Suppl 3):S145-S149

27 CDCSurgical site infection (SSI) event. Available at: http://www. cdc.gov/nhsn/pdfs/pscmanual/9pscssicurrent.pdf. Accessed January 25, 2017

28 Paek SH, Yun JY, Song SW, et al. The clinical impact of precise electrode positioning in STN DBS on three-year outcomes. J Neurol Sci 2013;327(1-2):25-31

29 Richardson RM, Ostrem JL, Starr PA. Surgical repositioning of misplaced subthalamic electrodes in Parkinson's disease: location of effective and ineffective leads. Stereotact Funct Neurosurg 2009;87(05):297-303

30 Cui $\mathrm{Z}$, Pan L, Song $\mathrm{H}$, et al. Intraoperative MRI for optimizing electrode placement for deep brain stimulation of the subthalamic nucleus in Parkinson disease. J Neurosurg 2016;124(01):62-69

31 Lanotte MM, Rizzone M, Bergamasco B, Faccani G, Melcarne A, Lopiano L. Deep brain stimulation of the subthalamic nucleus: anatomical, neurophysiological, and outcome correlations with the effects of stimulation. J Neurol Neurosurg Psychiatry 2002;72 (01):53-58

32 Asha MJ, Kausar J, Krovvidi H, et al. The effect of dopaminergic therapy on intraoperative microelectrode recordings for subthalamic deep brain stimulation under GA: can we operate on patients "on medications"? Acta Neurochir (Wien) 2016;158 (02):387-393 
33 Hertel F, Züchner M, Weimar I, et al. Implantation of electrodes for deep brain stimulation of the subthalamic nucleus in advanced Parkinson's disease with the aid of intraoperative microrecording under general anesthesia. Neurosurgery 2006;59(05):E1138, discussion E1138

34 Blume J, Schlaier J, Rothenfußer E, et al. Intraoperative clinical testing overestimates the therapeutic window of the permanent DBS electrode in the subthalamic nucleus. Acta Neurochir (Wien) 2017;159(09):1721-1726

35 Butson CR, McIntyre CC. Role of electrode design on the volume of tissue activated during deep brain stimulation. J Neural Eng 2006; 3(01):1-8

36 Schlaier JR, Habermeyer C, Janzen A, et al. The influence of intraoperative microelectrode recordings and clinical testing on the location of final stimulation sites in deep brain stimulation for Parkinson's disease. Acta Neurochir (Wien) 2013;155(02):357-366
37 O'Gorman RL, Jarosz JM, Samuel M, Clough C, Selway RP, Ashkan K. $\mathrm{CT} / \mathrm{MR}$ image fusion in the postoperative assessment of electrodes implanted for deep brain stimulation. Stereotact Funct Neurosurg 2009;87(04):205-210

38 Holl EM, Petersen EA, Foltynie T, et al. Improving targeting in image-guided frame-based deep brain stimulation. Neurosurgery 2010;67(2, Suppl Operative):437-447

39 Starr PA, Martin AJ, Ostrem JL, Talke P, Levesque N, Larson PS. Subthalamic nucleus deep brain stimulator placement using high-field interventional magnetic resonance imaging and a skull-mounted aiming device: technique and application accuracy. J Neurosurg 2010;112(03):479-490

40 Fenoy AJ, Simpson RK Jr. Risks of common complications in deep brain stimulation surgery: management and avoidance. J Neurosurg 2014;120(01):132-139 